\title{
The Influence of Binaural Meditative Sounds on Menses-Prosex Processes
}

\author{
Gabriela Iorgulescu*, Liliana Neagu \\ *MDD, MA, BA, PhD \\ Carol Davila University of Medicine and Pharmacy, Bucharest, Romania
}

\begin{abstract}
Though the benefits of music have been recognized since ancient times, the muzicoterapiei remains unexplored as is proper in the sense of knowledge influence on various bodily functions as well as its use in the treatment or regulation of physiological, cognitive, affective and behavioral processes.
\end{abstract}

Although many researchers have made their contribution in this respect, the scientific results are amazed every year by new discoveries made in laboratories or clinical halls, presented at numerous international conferences and symposia, thus highlighting the psychosomatic impact of music determined by various functions (emotional, cognitive, physiological, pedagogical, social, therapeutic and entertainment).

Our study aimed to know the influence of meditative binaural sounds on academic performance, the results reveal that they inhibit the ability to concentrate and learn (work memory), diminish resistance to effort and generate psychosomatic discomfort. However, recording and storing images (visual memory) seems to be facilitated in the ultra-relaxing meditation state, confirming studies that claim that during the memorization of images, intense theta activity is recorded, and they are associated with deep meditation.

KEYWORDS: binaural sounds, brain waves, attention, memory, brain stimulation

\section{INTRODUCTION}

The music is a form of expression that uses the sound but does not require words. No one knows if music was invented before language, after language or in the same time. Both are expressions of a general capacity for sophisticated cultural expression. Certain regions of the brain are used in both language and music. But music also uses parts of the brain that are not used in language, which means that music has unique functions, and at least, to a certain extent, a distinct evolutionary history - perhaps even one that precedes Homo Sapiens.

Regardless of its origin, music is present in every human culture, which means that a biological capacity for music evolved early in the history of our species. People are musicians by their nature - human DNA and the human brain give rise to our musical skills, so it is no surprise that the music is universally valid among human cultures. Whether you are listening to opera or rock, the ears translate the sound waves into nerve impulses in the brain. Music can activate many different areas of the brain, including regions responsible for emotions, for recognition of musical notes and rhythm.

The role of brain hemispheres in the perception of music Right brain hemisphere - passive (emotional) listening of music induces a dominant activity of the right hemisphere. Warner, 1999 (1) limits the involvement of the right hemisphere in the perception of music, only to the melody and voice, both having a broad emotional resonance. For this emotion generated by music there is a morpho-functional substrate in the nerve paths which leaves from the right hemisphere to the limbic system and the hypothalamus, from the base of the encephalus.
Left brain hemisphere - analyzes the frequency (height) of the sounds and the rhythm of the music. At the same time, the left auditory cortex interprets the meaning of words, their sequence, analyzes the message (being considered the seat of speech (Broca centers). Also at the level of this hemisphere are located the cortical areas involved in identifying the structure of a composition.

For musicians who have "absolute hearing," MRI has highlighted the involvement of the same areas of language as the headquarters of this rare ability. According to Sancar (2), people with absolute hearing have larger dimensions of the temporal zone, responsible for deciphering all musical notes.

Is the musical talent innate or can be learned? Scientists agree with both variants, at least in the case of musical ability: the absolute musical ear, the ability to identify a musical tone without comparing it with other tones. The absolute musical ear is usually found in family members, so scientists believe it has a genetic component. But early musical training is needed to develop this feature. Most musicians with an absolute musical ear began musical training before the age of four. After about 12 years it is impossible to develop an absolute musical ear.

Hemispheric interdependence - is a natural phenomenon, with the role of suppressing inhibited activity or become impossible by lesion, for example, from the contra lateral hemisphere. Also, this interdependence is necessary, whereas the analysis of information about the interval between sounds (the left hemisphere) is possible by processing the song by an intact structure (the right hemisphere). 


\section{The cortical and cortico-subcortical areas}

Cortico-thalamic projections ensures the stability of auditory images, while projections cortico-limbic, as well as the temporal lobe connections with the hippocampus, gives the listener the emotional tonus concurrently with the decoding of the musical message (3).

At limbic system level occurs the emotional-instructivebehavioral integration with natural, physiological, biorhythmic elements, which explains the influence of music on fundamental biorhythms responsible for adrenal cortical activity, circadian rhythm, rhythmic activity of respiratory and cardiac centers (4). Besides other elements included in the basic structures of the music (intensity, melody, soundtrack), the rhythm has direct influences on the limbic system (the connections of the acoustic sensory pathways with the limbic system), or by activating the common neural pathways between the areas of the temporal auditory cortex and the hippocampus, developing the ideative capacity of musicians and meloman, to whom the frequent listening or interpretation of complex music can induce the formation of biochemical links or internaluronal configurations involved in the process of thinking (5).

\section{The neuro-hormonal effects produced by music}

Studies have shown that different types of music induce different changes in the neuroendocrine system. McKinney et al., 1994 (6), conducted a study on a batch of 78 students, exploring the effect of music on $\beta$-endorphins. The results have shown that music can lower peripheral $\beta$-endorphin levels in healthy subjects, but in depressed patients the level of $\beta$-endorphins did not change, compared to the controlgroup, although they were significantly less depressed Another study (7) used two different types of music (techno and classical). A significant increase of heart rhythm, systolic blood pressure, $\beta$-endorphins, ACTH, norepinephrine and growth hormone was recorded in techno music, instead, classical music induced an improvement in emotional state, but no significant change in hormonal concentration.

Also, it has been found that pleasant music can regulate or affect various brain functions through dopaminergic neurotransmission and could be effective in rectifying symptoms in various diseases involving the dopamine secretion dysfunction. The musical audition can significantly increase plasma calcium levels and dopamine levels from neostriatal (calcium contributes to the synthesis of dopamine in the brain, causing a reduction in blood pressure (8)). Regarding the level of serotonin, studies have shown that, in general, the rhythmic music is the one that raises this level. Serotonin is a neurotransmitter involved in transmitting nerve impulses that help maintain feelings of joy. When the brain produces serotonin, the tension is diminished. Depression is a consequence of limited production of this hormone.

The study conducted by Evers and Suhr, 2000 (9), revealed increased release of 5-HT during pleasant music audition. Another study by Kumar et al, 1999 (10), investigated the effects of music concerning the concentrations of melatonin, norepinephrine, epinephrine, serotonin and prolactin in the blood in a group of patients with Alzheimer's disease. The results showed that the concentration level of melatonin in the serum has increased significantly at 6 weeks after the musical audition, helping to maintain the relaxed and calm mood of the patients. The levels of noradrenaline and adrenaline increased during the musical audition, but returned to the previous levels 6 weeks after music therapy. The serum concentration of serotonin, prolactin and prothrombin remained unchanged after 4 weeks of music therapy and at 6 weeks after the experiment.

Music is considered an important generator of eustres, and this positive reaction of the body is compared with a catecholamine reaction (11). Verle and Bell (12) reported the increase of catecholamines (adrenaline in particular) during rock concerts, where the particularity of music consists of rhythm and voice, having an exciting character and fast tempo. It should be noted that various types of music induce tinted reactions to the listener, inclusive to the healthy subject compared to the sick one, the music also having a regulator character - to increase or decrease the level of catecolamines under the influence of music. For example, to a stressed individual with an increased secretion of catecholamines, music can have a beneficial effect by lowering the serum levels of these hormones, and vice versa, to a bored and depressed individual it can grow. It is also important that the overcooling of the catecholamine reaction in techno or rock music can have harmful effects on cardiac patients. In an experiment conducted by McKinley et al. (6), in the case of some healthy subjects, the cortisol levels decreased after hearing some songs, along with mood improvement.

In hypertensive patients, salivary cortisol decreased after listening to the musical pieces. The contribution of hormonal dosages (salivary cortisol) and EEG records may surprise a turn to a positive mood before the appearance of clinical signs. In an experimental study conducted at Dr. Dan Prelipceanu Clinic, 2002 (13), was followed the psychological reaction and the cortisol reaction (salivary) of some depressed patients who listened to some contrasting fragments (slow-meditative music versus tumultuous rapid music). There has been an increase in salivary cortisol in 11 out of 12 patients, after listening the slow fragments, instead, the listening of stimulating music, was favorably evaluated by all patients, and serum cortisol values decreased in most patients ( 8 out of 12). In conclusion, it can be said that music can have not only favorable effects but can also be rejected, especially when we work with sick patients.

\section{The brain waves and the influence of different} frequencies on brain activity

The human brain continually produces brainwaves that measure in amplitude - the power of the electrical impulse (microvolts) and the frequency - the speed of electric waves $(\mathrm{Hz})$. Depending on the frequency, the brain wave categories are determined.

Beta waves (14-23 Hz) - are associated with the state of active attention and logical thinking. A prolonged activity of beta waves can generate panic states.

Alpha waves (8-14 Hz) - are associated with the installation of the relaxation state, while man can use his imagination and visualization capability. Alpha waves create a bridge between conscious and subconscious, in some cases materializing in states of meditation with a tendency to escape from ordinary reality.

Theta waves (4-8 Hz) - present in stages 1-2 of sleep and deep meditation, in association with a decrease of muscle tone. These are associated with the subconscious, behind 
them being "buried" memories, beliefs and emotions that govern our attitudes, hopes, behaviors. Also, profound imaginative experiences, such as dreams and visions, can materialize in frequent meditation states and a tendency to escape from ordinary reality, theta level being also related to the phenomenon of healing.

Delta waves $(0.5$ - $4 \mathrm{~Hz})$ - occur in stages 3-4 of sleep. The delta waves make the transition from superficial sleep to deep sleep, without eye movements and low muscle tone, being associated with the unconscious.

Stage 5 - is called paradoxical sleep, because although the subject sleeps with dreams and is totally relaxed, brain electrical activity is similar to the waking state. This is called REM sleep (Rapid Eye Movement). This stage occupies approx. $20 \%$ of the total night sleep and occurs discontinuously in 5-6 cycles lasting between 5-20 minutes. On the EMG, rapid eyeball movements occur, at this stage occurring dreams, as well as changes in heart rate and respiratory rate. This period is called the oniric phase, on the EEG being characteristic the sharp waves of theta band, recorded in the vertex region (14).

Between the bioelectric activity of the brain and the psychic activity there is a connection but insufficiently studied. Dr Becker's explorations have highlighted (15) that state of conscious attention is a function of electric currents, which moves between the two poles of the brain, negative and positive, so a real cerebral flow back and forth. If this electric flow is reversed we lose consciousness. Under the effect of a chemical anesthetic, the state of consciousness is reversed. The same effect have the acupuncture anesthesia, hypnosis, magnetic fields, autosugestia - all modify the electrical potentials of the body in the same way. Becker confirmed that under hypnosis, relaxation or visualization, change, in fact, the electrical potentials of the body. Dr. Leonard Ravitz (16) found that mind, memory, emotions - operate through a sophisticated electric circuit.

Neuroscience specialist Ueli Rutishauser, from Haward Hughes Medical Institute, California (17), together with his team, researched cellular processes at the hippocampus, recording the activity of nerve cells in the brain while people assimilated and updated information using sensitive electronic equipment, they registered an intense activity of theta waves, when the subjects memorized the images, but besides these findings, they noticed something that could anticipate the reminders in about a fifth of the cells, concluding that neural rhythms can sometimes be concerted, and this synchronicity helps people form persistent memories. In the learning phase, the team noticed that the patients had a good chance of remembering the images if they were run at a time when the potentials of action of the hippocampus and the amygdala nuclei synchronized with theta theta waves, recorded locally. The study highlights the existence of emotional impact, out of focus, which makes certain things to be memorized, namely, synchronization.

Every state of consciousness in which a person is at any moment is a combination of the four categories of brainwaves. The extraordinary states of lucidity are the result of the precise combination of all these types of waves. In solving some arithmetic problems, there is the phenomenon of spatial synchronization of the bioelectric processes, especially from certain points of the prefrontal region, in which are located the tertiary areas of the programming and regulation area; it is worth noting that the degree of spatial synchronization of biopotentials decreases or the phenomenon completely disappears in the case of the automation of intellectual activity (18).

\section{Types of brain stimulation}

When frequencies are used to synchronize the brain with a modified state, there are several possible strategies. Using different frequencies, it is possible to resonate various areas of the body, thus activating the emotional and mental states associated with those areas (19).

\section{Isocronic stimulation -}

is achieved by rhythm and constant stimuli or pulses, evenly distributed, which start and stop wirh a specified rate/second. Strong reaction in the brain is triggered by sounds that stop suddenly, this being the most effective form of stimulation, with different effects depending on waveforms (rectangular, triangular or sinusoidal).

\section{Binaural stimulation -}

is achieved by the "differential signaling" method in which two different tones are transmitted to the brain (eg. $200 \mathrm{~Hz}$ to an ear and $208 \mathrm{~Hz}$ to the other - the difference between the signals being $8 \mathrm{~Hz}$, and the brain will resonate at $8 \mathrm{~Hz}$ (binaural beating)). The effect of listening the binaural beats can be relaxation or stimulation. Binaural beats in the delta (1$4 \mathrm{~Hz}$ ) and theta (4-8 Hz) domains have been associated with relaxation, meditation and creativity (20). Binaural beats in the alpha domain $(8-12 \mathrm{~Hz}$ ) increased alpha waves associated with memory improvement (21). When the tones frequencies are spaced by $25 \mathrm{~Hz}$, the brain is no longer able to detect the relationship between them, so tones are perceived individually.

\section{Monoaural stimulation-}

although similar to binaural stimulation, battles can be produced at almost any frequency (even beyond $1 \mathrm{KHz}$ ) and can be perceived even if there is a separation between them of more than $25 \mathrm{~Hz}$, but to maintain the full effect, both tones must be on the same amplitude. Similar to binaural principles, they are distinguished by the fact that slightly different sounds are generated in each individual ear, as a result of the summation of two waveforms that are close, that they gather and fall with each other, being perceived as a pulse or beating as the binaural (22).

\section{Using music to increase cognitive performance}

The researches has shown that while listening to highfrequency music, there is some harmonization and synchronization of the cerebral hemispheres, generating an exceptional mental focus with the possibility of a potential mental reorganization in a higher order.

Dr. Aryel Routtenberg (23) was among the first to discover that electrical stimulation of certain areas of the brain releases neurotransmitters involved in memory, learning and creativity processes. Dr. Capel has discovered that different waves lead to the triggering of different neurotransmitters, such as: a $10 \mathrm{~Hz}$ signal - increases production of serotonin that induces calm and attenuates pain; the frequencies of 90$111 \mathrm{~Hz}$ produce endorphins, and those of $4 \mathrm{~Hz}$ catecholamines, important in the process of consolidating memory and attention (24). Dr. Alfred Tomatis (25) analyzed the influence of high-frequency sounds (5000-8000 Hz) and 
found that they produced the unlocking of emotional and cognitive blockages. According to studies, music rich in highfrequency sounds is the one composed by Mozart, stimulating the connections between neurons. Researchers have demonstrated an isomorphism between brain activity and the rhythm of Mozart's music (rises and decreases in sound intensity), which is repeated at 20-30 sec. and acting on neuronal groups, whose activities are also carried out in cycles of 30 seconds (26), generating a quasi-synchronous activation of a large number of cortical areas, making it possible to globalize cognitive processes. According to Sengewald (1992) and Wieser (2000), Mozart's music totally stimulates $(100 \%)$ the cerebral cortex, but also other musical creations may have the same effect (27).

An important aspect is the creation of a steady state in the functioning of cerebral hemispheres, these being complementary, and when its working together they produce the phenomenon of synergy. When the moments of maximum amplitude of the two waves of the cerebral hemisphere coincide, will produce a two-tier wave than the one at the beginning, so the cerebral hemispheres will operate synchronously and the amplitude of the brain waves in the entire cortex increases greatly, having the possibility of a potential mental reorganization in a higher order. Dr. Lester Fehni, says that the synchronization of brain hemispheres determines an extraordinary mental focus, with a growing of the power of awareness. The callous body containing axonal fibers that interconnects brain hemispheres is activated by music, which increases the ability of the two hemispheres to work in the unit, rather than in the opposition (28). An important aspect is that Soviet tests with magnetic or electric fields of extremely low frequency have demonstrated that by sequentially starting and stopping these fields it can lead to brain dishing, interfering with the hypothalamus, which is responsive to logical and associative thinking, also affecting acetylcholine - an important neurotransmitter in mnesic processes, at the same time acting on other important neurotransmitters in memory - norepinephrine and dopamine (29).

\section{Personal Research:}

1. The issue:

Improving intellectual performance is one of the main concerns of people, so finding some effective strategies to stimulate and overcome the limits is a fascinating field of interest of all times.

\section{The objective of research -}

is to find ways to improve cognitive performance with the help of sounds and to identify what kind of music can stimulate or, on the contrary, inhibit mental and prosexic capabilities.

\section{Hypothesis -}

Binaural sounds that perform ultra-relaxing meditation (monotone, repetitive, without a melodic line sounds) may have a stimulating or inhibiting effect on memory and attention.

\section{Research Method:}

A. Subjects - In the samples used 264 students were included, 2nd year in Dental Medicine, within the U.M.F. "Carol Davila" Bucharest, for testing concentrated attention and visual memory, divided into two equal groups of 132 students, both for the experimental group and for the control group, and for contextual memory testing 216 students were included, divided into 2 equal groups: as many as 108 students for the two groups control and experimental, the lots assuring sufficient statistical power to identify the significance threshold difference of 0.05 .

B. Tools and materials: In research the data collection method was mainly based on standardized samples that lead to numerical data. All study participants were evaluated before and after the application of independent variables, at an interval of one week.

For dependent variables, the tools used to evaluate the results were as follows:

$>$ Working memory._contextual) was appreciated using 4 courses from the "Genetics" discipline, of which 6 questions from each course were formulated, with 1 point being scored the correct answers; time used - 45 minutes.

$>$ Visual memory_was measured using the Ray-Claparedé test, comprising 30 images, of which 15 images were used before and 15 after applying the independent variable; application time - 10 minutes.

$>$ Concentrated attention_- measured by the ToullousePieron test, which contains 1000 graphic signs (of which 378 are significant stimuli); execution time - 10 minutes. The test refers to the coefficient of operability (total number of marks traveled), the coefficient of accuracy (number of omissions recorded) and the coefficient mnezic (number of errors).

For the independent variable we used binaural sounds Ultra Deep Meditation - Binaural Beats (369: 14 - MB) listening to headphones.

5. The design used is an experimental one and includes the following variables:

A. the independent variable -is represented by binaural sound intervention;

B. dependent variables - are those to which the expected effect relates, namely: visual memory, contextual memory and concentrated attention.

\section{Procedure:}

Students received instructions for the use of tests and execution time. Measurements were made before and after the introduction of the independent variables, one week between the test and the retest, to test concentrated attention and visual memory for both control and experimental groups (who listened to binaural sounds during retesting). For contextual memory, students in the experimental group listened to binaural sounds during material learning, and students in the control group have taught the courses without sound background.

\section{Data Analysis -}

Considered the extent to which response levels change significant or not, after listening the binaural sounds. For the verification, the T-test was used for the difference between the averages of two dependent samples which allows the assessment of the significance of the variation of a particular characteristic to the same subjects in two different situations ("before" and "after" the action of a particular condition) or in two different contexts, regardless of the time of their occurrence. 


\section{Results:}

1. Experimental model (contextual memory)

The results obtained at each course.

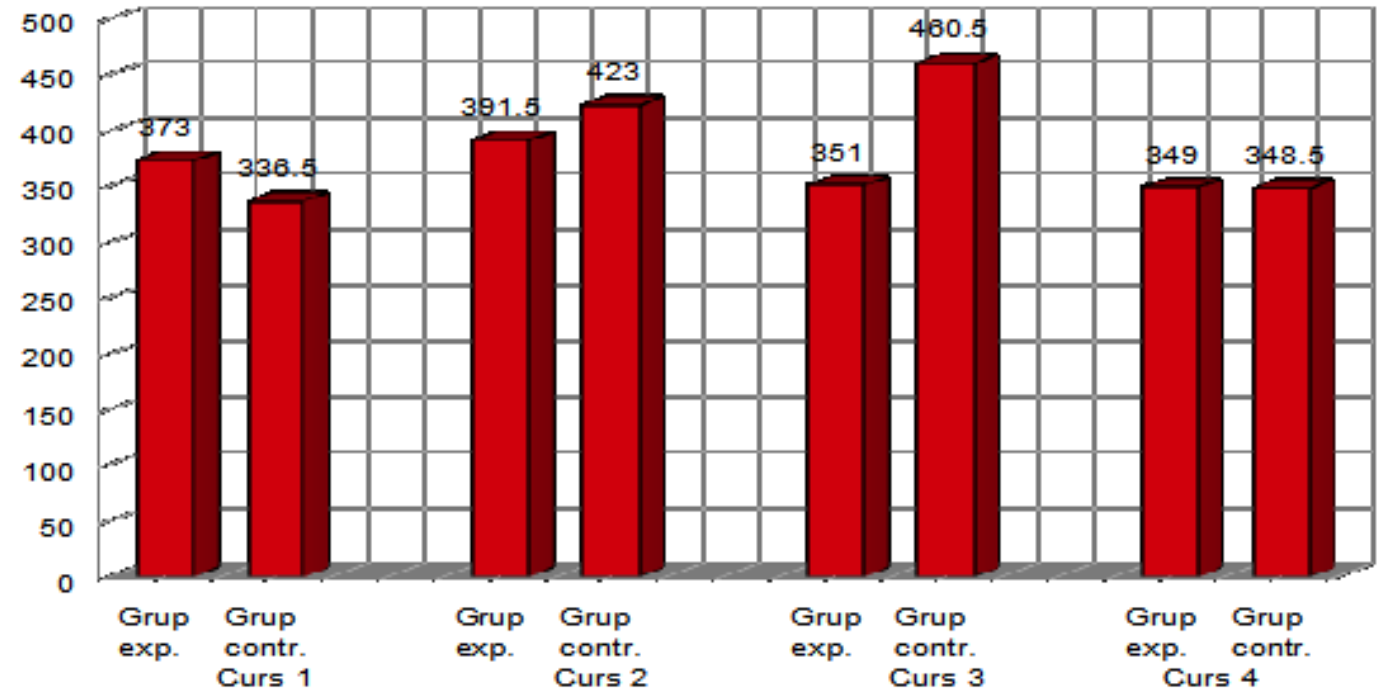

The control group obtained a higher overall score of 104 points compared to the experimental group.

1. Compilation of samples

A. The first sample - $\mathbf{e} \mathbf{1}$ considered experimental group, is a

where $\mathrm{N}=$ number of subjects group of 108 students who participated in courses listening the binaural sounds

B. The second sample - $\mathbf{e} 2$ considered a control group, is a group of 108 students who participated in classes

without listening any kind of music. $\mathrm{df}=$ degrees of freedom

SS $=$ standard error of difference

$\mathrm{s} 2=$ dispersion

The T test value

Students from each group attended a number of four courses, and after each course they were subjected to a test consisting of 6 questions. The grades obtained at the courses were $s_{p}^{2}=\left(\left(d f_{1} /\left(d f_{1}+d f_{2}\right)\right) * s_{1}^{2}\right)+\left(\left(d f_{2} /\left(d f_{2}+d f_{2}\right)\right) * s_{2}^{2}\right)=$ $((107 / 214) * 12.15)+((107 / 214) * 12.78)=12.47$ summed up.

\begin{tabular}{|c|c|c|c|}
\hline Group & $\begin{array}{c}\text { Number of } \\
\text { subjects }\end{array}$ & Mean & $\begin{array}{c}\text { Mean } \\
\text { difference }\end{array}$ \\
\hline Experimental & 108 & 13,60 & -0.75 \\
\hline Control & 108 & 14,36 & \\
\hline
\end{tabular}

To demonstrate the significance of the difference between the two groups, we used the T test for two independent samples.

\section{Differences calculations:}

Experimental group

$N_{1}: 108$

$d f_{1}=N-1=108-1=107$

$M_{1}: 13.61$

$S S_{1}: 1300.03$

$S_{1}^{2}=S S_{1} /(N-1)=1300.03 /(108-1)=12.15$

\section{Control group}

$N_{2}: 108$

$d f_{2}=N-1=108-1=107$

$M_{2}: 14.36$

$S S_{2}: 1367.92$

$S_{2}^{2}=S S_{2} /(N-1)=1367.92 /(108-1)=12.78$

${ }_{M 1}^{2}=s_{p}^{2} / N_{1}=12.47 / 108=0.12$

$s 2 M_{2}=s 2 p / N 2=12.47 / 108=0.12$

$t=(M 1-M 2) / \sqrt{ }\left(s 2 M_{1}+s 2 M_{2}\right)=-0.75 / \sqrt{ } 0.23=-1.57$

The $t$ value is $\mathbf{- 1 . 5 7 0 5 4}$. The $p$ value is 0.117767 . The result is insignificant if $p<0.05$.

The means obtained by the two groups Mediile obtinute de cele doua grupuri

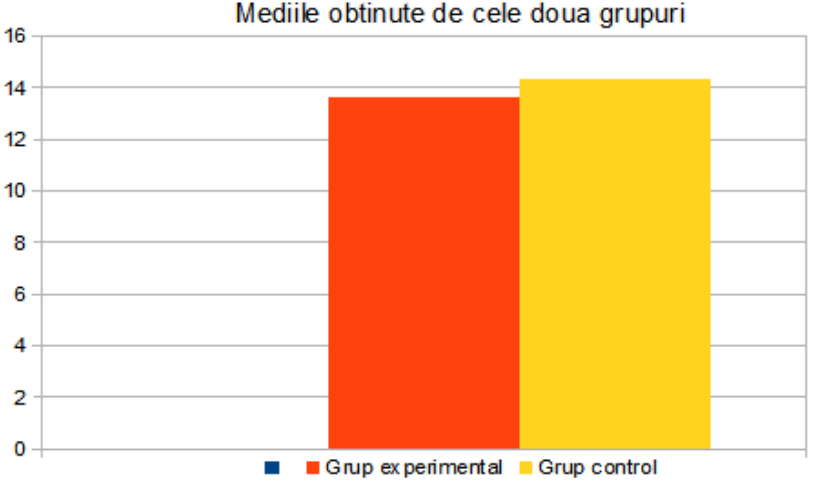

2. Experimental model (concentrated attention and visual memory]

1. Compilation of samples

A. The first sample - e1 considered experimental group, is a group of 132 students who attended courses listening the binaural sounds. 
International Journal of Trend in Scientific Research and Development (IJTSRD) @ www.ijtsrd.com eISSN: 2456-6470

B. The second sample - e2 considered a control group, is a group of 132 students who attended classes without

listening any kind of music.

Experimental group

To demonstrate the significance of the difference between before and after we used the T test for two dependent samples.

Concentrated attention

\begin{tabular}{|c|c|c|c|}
\hline & Number of signs traveled & omissions & errors \\
\hline $\begin{array}{l}\text { Differences } \\
\text { calculations }\end{array}$ & $\begin{array}{c}\text { Media: } 163.38 \\
\mu=0 \\
S^{2}=S S / d f=1518335.06 /(132-1) \\
=11590.34 \\
S_{M}^{2}=S^{2} / N=11590.34 / 132= \\
S_{M}=\sqrt{S_{M}^{2}}=\sqrt{87.81}\end{array}$ & $\begin{array}{c}\text { Media: }-1.95 \\
\mu=0 \\
S^{2}=S S / d f=22358.63 /(132-1)= \\
170.68 \\
S_{M}^{2}=S^{2} / N=170.68 / 132= \\
1.29 \\
S_{M}=\sqrt{S_{M}^{2}}=\sqrt{1.29}=1.14\end{array}$ & $\begin{array}{c}\text { Media: } 0.16 \\
\mu=0 \\
S^{2}=S S / d f=237.66 /(132-1)= \\
1.81 \\
S_{M}^{2}=S^{2} / N=1.81 / 132=0.01 \\
S_{M}=\sqrt{S}_{M}^{2}=\sqrt{0.01}=0.12\end{array}$ \\
\hline $\begin{array}{l}\text { The T test } \\
\text { value }\end{array}$ & $\begin{array}{c}t=(M-\mu) / S_{M}=(163.38- \\
0) / 9.37=17.44\end{array}$ & $\begin{array}{c}t=(M-\mu) / S_{M}=(-1.95-0) / 1.14 \\
=-1.71\end{array}$ & $\begin{array}{c}t=(M-\mu) / S_{M}=(0.16-0) / 0.12= \\
1.36\end{array}$ \\
\hline $\begin{array}{l}\text { Significance } \\
\text { of the test }\end{array}$ & $\begin{array}{l}\text { The value of } t \text { is } 17.435503 \text {. The } \\
\text { value of } p \text { is }<0.00001 \text {. The } \\
\text { result is significant at } \mathrm{p} \leq 0.05 \text {. }\end{array}$ & $\begin{array}{c}\text { The value of } t \text { is }-1.712219 \text {. The } \\
\text { value of } p \text { is } 0.089223 \text {. The } \\
\text { result is not significant at } \mathrm{p} \leq \\
0.05 \text {. }\end{array}$ & $\begin{array}{l}\text { The value of } t \text { is } 1.357034 \text {. The } \\
\text { value of } p \text { is } 0.177104 \text {. The result } \\
\text { is not significant at } \mathrm{p} \leq 0.05 \text {. }\end{array}$ \\
\hline
\end{tabular}

\section{Visual memory \\ Differences calculations}

Mean: 0.20

$\mu=0$

$S^{2}=S S / d f=583.48 /(132-1)=4.45$

$S_{M}^{2}=S^{2} / N=4.45 / 132=0.03$

$S_{M}=\sqrt{ } S_{M}^{2}=\sqrt{0.03}=0.18$

\section{The T test value}

$t=(M-\mu) / S_{M}=(0.20-0) / 0.18=1.11$

The value of $t$ is 1.113526 . The value of $p$ is 0.267521 . The result is not significant at $\mathrm{p} \leq 0.05$.

\section{Control group}

To demonstrate the significance of the difference between before and after we used the T test for two dependent samples.

\begin{tabular}{|c|c|c|c|}
\hline & Number of signs traveled & omissions & errors \\
\hline $\begin{array}{l}\text { Differences } \\
\text { calculations }\end{array}$ & $\begin{array}{c}\text { Media: } 225.31 \\
\mu=0 \\
S^{2}=S S / d f=1883606.27 /(132- \\
1)=14378.67 \\
S_{M}^{2}=S^{2} / N=14378.67 / 132= \\
108.93 \\
S_{M}=\sqrt{S_{M}^{2}}=\sqrt{108.93=10.44}\end{array}$ & $\begin{array}{c}\text { Media: }-1.66 \\
\mu=0 \\
S^{2}=S S / d f=65703.66 /(132-1)= \\
501.55 \\
S_{M}^{2}=S^{2} / N=501.55 / 132=3.80 \\
S_{M}=\sqrt{S_{M}^{2}}=\sqrt{3.80}=1.95\end{array}$ & $\begin{array}{c}\text { Media: } 0.39 \\
\mu=0 \\
S^{2}=S S / d f=519.30 /(132-1)= \\
3.96 \\
S_{M}^{2}=S^{2} / N=3.96 / 132=0.03 \\
S_{M}=\sqrt{S_{M}^{2}}=\sqrt{0.03=0.17}\end{array}$ \\
\hline $\begin{array}{c}\text { The T test } \\
\text { value }\end{array}$ & $\begin{array}{c}t=(M-\mu) / S_{M}=(225.31- \\
0) / 10.44=21.59\end{array}$ & $\begin{array}{c}t=(M-\mu) / S_{M}=(-1.66-0) / 1.95= \\
-0.85\end{array}$ & $\begin{array}{c}t=(M-\mu) / S_{M}=(0.39- \\
0) / 0.17=2.23\end{array}$ \\
\hline $\begin{array}{l}\text { Significance } \\
\text { of the test }\end{array}$ & $\begin{array}{l}\text { The value of } t \text { is } 21.587840 \text {. The } \\
\text { value of } p \text { is }<0.00001 \text {. The } \\
\text { result is significant at } \mathrm{p} \leq 0.05 \text {. }\end{array}$ & $\begin{array}{c}\text { The value of } t \text { is }-0.851134 \text {. The } \\
\text { value of } p \text { is } 0.396248 \text {. The result } \\
\text { is not significant at } \mathrm{p} \leq 0.05 \text {. }\end{array}$ & $\begin{array}{l}\text { The value of } t \text { is } 2.229521 \text {. The } \\
\text { value of } p \text { is } 0.027482 \text {. The } \\
\text { result is significant at } \mathrm{p} \leq 0.05 \text {. }\end{array}$ \\
\hline
\end{tabular}


International Journal of Trend in Scientific Research and Development (IJTSRD) @ www.ijtsrd.com eISSN: 2456-6470

Visual memory

Differences calculations

Mean: 0

$\mu=0$

$S^{2}=S S / d f=0 /(132-1)=0$

$S_{M}^{2}=S^{2} / N=0 / 132=0$

$S_{M}=\sqrt{ } S_{M}^{2}=\sqrt{ } 0=0$

The T test value

$t=(M-\mu) / S_{M}=(0-0) / 0=0$

The value of $t$ is 0 . The value of $p$ is 1 . The result is not significant at $\mathrm{p} \leq 0.05$.

Differences between experimental and control groups To demonstrate the significance of the difference between before and after the two groups we used the T test for two independent samples.

\section{Concentrated attention}

Number of signs traveled

\section{Differences calculations}

Experimental group

$N_{1}: 132$

$d f_{1}=N-1=132-1=131$

$M_{1}: 163.38$

$S S_{1}: 1518335.06$

$s_{1}^{2}=S S_{1} /(N-1)=1518335.06 /(132-1)=11590.34$

\section{Control group}

$N_{2}: 132$

$d f_{2}=N-1=132-1=131$

$M_{2}: 225.31$

$S S_{2}: 1883606.27$

$S_{2}^{2}=S S_{2} /(N-1)=1883606.27 /(132-1)=14378.67$

\section{The T test value}

$s_{p}^{2}=\left(\left(d f_{1} /\left(d f_{1}+d f_{2}\right)\right) * s_{1}^{2}\right)+\left(\left(d f_{2} /\left(d f_{2}+d f_{2}\right)\right) * s_{2}^{2}\right)=$ $((131 / 262) * 11590.34)+((131 / 262) * 14378.67)=$ 12984.51

${ }^{2}{ }_{M 1}={ }^{2}{ }_{p} / N_{1}=12984.51 / 132=98.37$

$s 2 M_{2}=s 2 p / N 2=12984.51 / 132=98.37$

$t=(M 1-M 2) / \sqrt{ }\left(s 2 M_{1}+s 2 M_{2}\right)=-61.93 / \sqrt{ } 196.73=-4.42$

The $t$-value is -4.41543 . The $p$-value is $<.00001$. The result is significant at $p<.05$.

\section{The effect size}

Cohen's $d=(225.31-163.38) / 1710747.312647=$ 0.000036 .

Gates' delta $=(225.31-163.38) / 1518335=0.000041$.
Hedges' $g=(225.31-163.38) / 1710747.312647=$ 0.000036 .

The concentrated attention - Number of signs traveled (mean)

Atentia concentrata

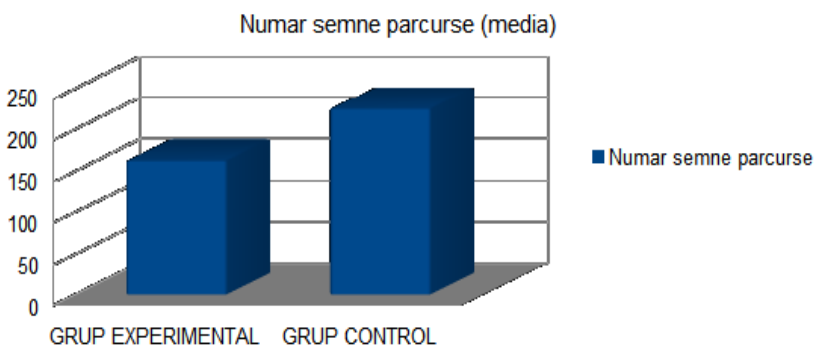

\section{Omisiuni}

\section{Differences calculations}

Experimental group

$N_{1}: 132$

$d f_{1}=N-1=132-1=131$

$M_{1}:-1.95$

$S S_{1}: 22358.63$

$S_{1}^{2}=S S_{1} /(N-1)=22358.63 /(132-1)=170.68$

Control group

$N_{2}: 132$

$d f_{2}=N-1=132-1=131$

$M_{2}:-1.66$

$S S_{2}: 65703.66$

$S_{2}^{2}=S S_{2} /(N-1)=65703.66 /(132-1)=501.55$

The $\mathrm{T}$ test value

$s_{p}^{2}=\left(\left(d f_{1} /\left(d f_{1}+d f_{2}\right)\right) * s_{1}^{2}\right)+\left(\left(d f_{2} /\left(d f_{2}+d f_{2}\right)\right) * s_{2}{ }_{2}\right)=$ $((131 / 262) * 170.68)+((131 / 262) * 501.55)=336.12$

$s_{M 1}^{2}=s_{p}^{2} / N_{1}=336.12 / 132=2.55$

$s 2 M_{2}=s 2 p / N 2=336.12 / 132=2.55$

$t=(M 1-M 2) / \sqrt{ }\left(s 2 M_{1}+s 2 M_{2}\right)=-0.29 / \sqrt{5} .09=-0.13$

The $t$-value is -0.12757 . The $p$-value is .449295 . The result is not significant at $p<.05$.

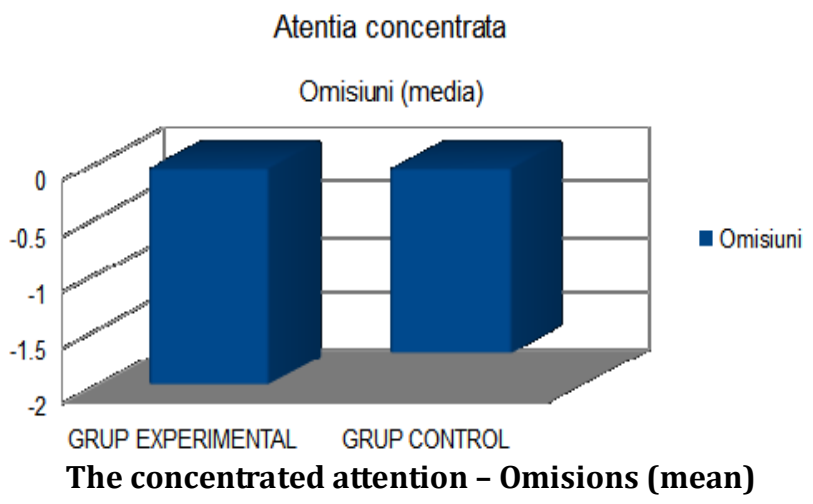


Differences calculations

Errors

Experimental group

$N_{1}: 132$

$d f_{1}=N-1=132-1=131$

$M_{1}: 0.16$

$S S_{1}: 237.66$

$s_{1}^{2}=S S_{1} /(N-1)=237.66 /(132-1)=1.81$

Control group

$N_{2}: 132$

$d f_{2}=N-1=132-1=131$

$M_{2}: 0.39$

$\mathrm{SS}_{2}: 519.3$

$S_{2}^{2}=S S_{2} /(N-1)=519.3 /(132-1)=3.9$
$M_{2}:-1.07$

$\mathrm{SS}_{2}: 498.39$

$s_{2}^{2}=S S_{2} /(N-1)=498.39 /(132-1)=3.8$

\section{The $\mathrm{T}$ test value}

$s_{p}^{2}=\left(\left(d f_{1} /\left(d f_{1}+d f_{2}\right)\right) * s_{1}^{2}\right)+\left(\left(d f_{2} /\left(d f_{2}+d f_{2}\right)\right) * s_{2}^{2}\right)=$

$((131 / 262) * 4.45)+((131 / 262) * 3.8)=4.13$

$s_{M 1}^{2}=s_{p}^{2} / N_{1}=4.13 / 132=0.03$

$s 2 M_{2}=s 2 p / N 2=4.13 / 132=0.03$

$t=(M 1-M 2) / \sqrt{ }\left(s 2 M_{1}+s 2 M_{2}\right)=1.27 / \sqrt{ } 0.06=5.09$

The $t$-value is 5.08829 . The $p$-value is $<.00001$. The result is significant at $p<.05$.

\section{The effect size}

Cohen's $d=(-1.07-0.2) / 542.605521=0.002341$.

Gates' delta $=(-1.07-0.2) / 583.48=0.002177$.

Hedges' $g=(-1.07-0.2) / 542.605521=0.002341$.

The $\mathrm{T}$ test value

$s_{p}^{2}=\left(\left(d f_{1} /\left(d f_{1}+d f_{2}\right)\right) * s_{1}^{2}\right)+\left(\left(d f_{2} /\left(d f_{2}+d f_{2}\right)\right) * s_{2}^{2}\right)=$

$((131 / 262) * 1.81)+((131 / 262) * 3.96)=2.89$

$s_{M 1}^{2}=s_{p}^{2} / N_{1}=2.89 / 132=0.02$

$s 2 M_{2}=s 2 p / N 2=2.89 / 132=0.02$

$t=(M 1-M 2) / \sqrt{ }\left(s 2 M_{1}+s 2 M_{2}\right)=-0.23 / \sqrt{ } 0.04=-1.09$

The $t$-value is -1.08626 . The $p$-value is 13918 . The result is not significant at $p<.05$.

\section{The concentrated attention - errors (mean)}

Atentia concentrata

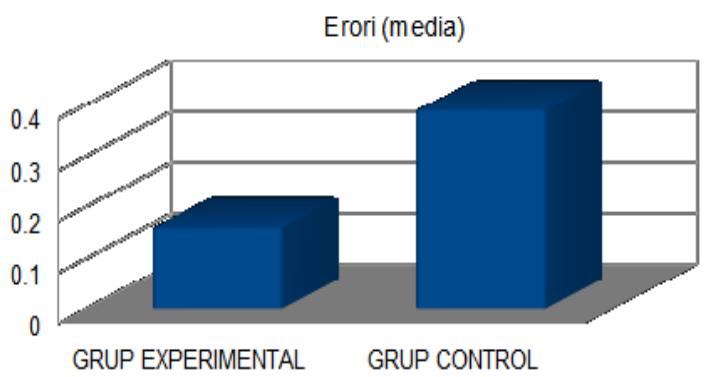

Visual memory

\section{Differences calculations}

Experimental group

$N_{1}: 132$

$d f_{1}=N-1=132-1=131$

$M_{1}: 0.2$

$S S_{1}: 583.48$

$S_{1}^{2}=S S_{1} /(N-1)=583.48 /(132-1)=4.45$

\section{Control group}

$N_{2}: 132$

$d f_{2}=N-1=132-1=131$

\section{The visual memory}

Memoria vizuala

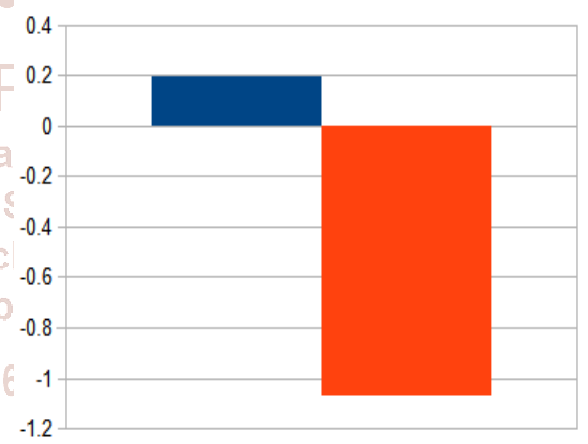

- GRUP EXPERIMENTAL - GRUP CONTROL

\section{Discutions:}

According to research in the field of music therapy, in terms of the influence of music on menses and prosexic processes we can say that the sound background changes the material substrate of memory and the electrical activity of the brain, but which of these favors cognitive processes?

Research shows that after listening to high-frequency music for a certain amount of time, the mind is harmonizing, is sharpening, overcoming the difficulty of learning. The effect of "Mozart" is explained by the fact that the rhythm of his music reproduces the cyclical rhythms of the brain cells, stimulating the connections between the neurons. Dr. Lozanov discovered that music at a rate of 60 beats / minute increases alpha activity, associated with relaxation, favoring the overall improvement of memory. Eccles supports the hypothesis that a repeated excitation of the neuron leads to the emergence of new contact points, either with the dentures or the body of another cell, which would facilitate the circulation of the nervous influx and the possibility of reminding.

As for the attention, its focus is the result of the appearance in the cerebral cortex of an area of optimal excitability. The stimulation of reticulated formation leads to the appearance of the orientation reflex. This formation is closely related to 
the cortex. Based on the ascending reticulated system, the reticulated formation activates the cortex, in turn, the cortex acting downwardly, has an excitatory or inhibitory action on the reticulated formation. Reticular formation of the brainstem generates a tonic reaction, it alerts the cortex, while the thalamic projections of the reticulated system generate a phasic reaction involved in focusing and switching attention. A slight increase of the tense will determine the maximum operation. Amplification of mental alertness increases the ability to concentrate, this being essential to achieve maximum performance. On the other hand, in the case of people who are already very tense, an increase in alert status can lead to anxiety and, implicitly, to a decrease in performance. Concerning concentrated attention, the researchers have found that if they perform repetitive and monotonous tasks, the brain involuntarily falls into rest, no longer being responsive and efficient.

\section{Conclusions:}

A. According to the obtained results, the concentration and focusing capacity is reduced in the experimental group relative to the control group, but also with the results obtained before and after the introduction of the independent variable, for both the operability coefficient and the accuracy, in the sense that the students in the experimental group performs the attention test slower, recording at the same time, a greater number of failures, affecting the speed of the observation process (dynamic nerve processes) and resistance to disturbing factors. Instead, the number of errors is higher in the control group during the retest, which means that the durability of the operational scheme over time is kept in the experimental group, and the control group appears to have some disinterest in the execution of the tasks, affecting the duration of the learning period, productivity, ability to transfer operational schemes.

B. Also, in the case of contextual memory, there is a decrease in the performances of the experimental group compared to the control group.

C. As for visual memory, the results show that the scores obtained in the experimental group are better than the control, but without significant value. The visual material is stored in different areas from the verbal material, although the storage mechanisms are the same. We can conclude that binaural sounds act more on the sensory register, activating neurons in the visual cortex.

\section{Note:}

It should be noted that a total of 32 students reported general discomfort, headaches, dizziness, vomiting, drowsiness, restlessness, difficulty concentrating, irritability while trying to learn or perform the tests on the sound background used in the experiment, for example ("I experienced headaches", "the sounds disconnected me from learning", "during the ascension, the concentration was diminished, there were times when I had to stop in the middle of a word", "I felt a mental discomfort of a value of 2 on a scale of 0-5 when I had to focus, but if I listened to the sounds without having to do another action at the same time, then the discomfort is - 0 - sometimes can be relaxing". Most often they were mentioned the headaches.

As a general conclusion, we can say that binar sounds "Ultra Deep Meditation - Binaural Beats" prove to be ineffective in amplifying the performance of concentrated attention and working memory, on the contrary, they inhibit the ability to concentrate and diminish resistance to voluntary effort to maintain the stability and focus of attention, a fact that was also highlighted by subjects who experienced negative symptoms, such as those mentioned above. Repetitive and monotone sounds make the brain to get in a state of rest, those having an inhibitory action on the reticulated formation (not recommended for other tasks requiring concentration, such as driving), compared to rhythmic music (proven in the research presented), in which, under their influence, the reticulated formation from the brain stem generates a tonic reaction that alerts the cortex, increasing the concentration and orientation of nerve energy.

On the other hand, given that the binaural beats used in the study affects brain waves, realizing a deeply ultra-relaxing meditation could explain the slightly increased results of the experimental group in terms of visual memory, confirming somewhat the results presented by Ueli Rutishuser, like that during the memorization of images, an intense activity of theta waves was found, storing images being more faithful when action potentials of the hippocampus and the amygdala nuclei synchronize with theta waves, however, the results are not significantly different from the control group, and this must be demonstrated in other research in which to use meditative music, inducing the theta waves.

\section{REFERENCES}

[1] Warner, Nick and Victor Aziz (2005), Hymns and arias: Musical hallucinations in older people in Wales. International Journal of Geriatric Psychiatry 20: 638660 ;

[2] Sancar, F.- Music aand the brain; processing and responding (a general overview);

[3] Roesser \& Dali, cit. Iamandescu, I.B., Receptive Music Therapy, 2nd Edition, "Andrei Şaguna" Publishing House, Constanţa, 2011, pg.201;

[4] Luban-Plozza, B., Dalli Ponti, M., Dickhaut, H. (1988). Musik and Psyche, Birkhauser Tatgeber, Basel, Boston, Berlin;

[5] Sancar, F., Music and the brain: processing and responding (a general overview);

[6] McKinney, C.H., Antoni, M.H., Kumar, M. et al. (1997), Effects of guided imagery and musik (GIM) on mood and cortisol in healthy adults. Health Psychol., 16 (4): 390-400;

[7] Gerra G, Zaimovic A, Franchini D, Palladino M, Gincastro G., et al. (1998), Neuroendocrine responses of healthy volunteers to "techno-music": relationschips with personality traits and emotional state. In Psychophysiol 28: 99-111. PMID: 9506313;

[8] Sutto D, akiyama K (2004). Music improves dopaminergic neurotransmission demonstration on the effect of music on blood pressure regulation, Brain Research., 6; 1016 (2): 255-62;

[9] Evers S., Suhr B. (2000), Changes of the neurotransmitter serotonin but not of hormones during short time music perception, European Archives of Psychiatry and Clinical Neuroscience, vol. 250, Number 3, 144-147; 
[10] Kumar AM, Tims F, Cruess DG, Mintzer MF, Ironson G, Loewenstein D, Cattan R, Fernandez JB, Eisdorfer C, Kuman M. (1999), Music therapy increases serum melatonin levels in patients with Alzheimer's disease. Alternative Therapies in Health and Medicine: 5 (6): 49-57;

[11] Iamandescu, I.B., (2011) Receptive Music Therapy, 2nd Edition, "Andrei Şaguna" Foundation Publishing House, Constanţa;

[12] Verle and Bell, cit. Iamandescu, I.B., (2011), Receptive Music Therapy, 2nd edition, "Andrei Şaguna" Foundation Publishing House, Constanţa;

[13] Prelipceanu, D., (2000), Mental Health Treaty, Enciclopedic Publishing House, Bucharest;

[14] Boisteanu, D., Sleep Medicine, p.375.

[15] Becker, F., cit. Liliana Neagu (2011), The Quality and the Overall Effects of Music on the Development of Mind and Prosecutive Capacity", rev. Medical Practice, Vol. 6, No. 2 (22), pp. 144-149;

[16] Ravitz, L., cit. Liliana Neagu-Simion (2010), Influence of music on cognitive processes - theoretical basis of the use of music therapy in neurological and psychiatric disorders, vol. XVII, Nr. 2, pg.105-110;

[17] Rutishauser, U., Schuman, EM., Mamelak, AN (2006), J Neurosci Methods 154: 204-224;

[18] Purcia, D.C., Purcia, S., (2001), Neuropsychophysiology Problems, Ed. "Lucian Blaga" University, Sibiu;

[19] Internet Source: http / psychology: 50, webs: com / Melter: htm accessed: 29.01.2011;
[20] Hiew, C.C. (1995) - Hemi-Sync in creativity: Hemi-Sync Journal, XIII (1), pp.3-5;

[21] Kennerly, R.C. (1994). An empirical investigation into the effect of beta frequency binaural beat audio signals on four meures of human memory. (Department of Psychology, West Georgia College, Carrolton, Georgia;

[22] Internet Source: www.perfect_mind.com/tehnologiaperfect-mind.accesat 7.10.2018;

[23] Routtenberg, A., (1979). Anatomical and biochemical evidence. Pharmacology of Central Motivational Systems, Federation Proceedings. 38: 2446-2453;

[24] Internet Source: www.founmyfitness.com/episodes/pierre-capel;

[25] Tomatis, A., Internet Source: http://www.ncbi.nlm.nih.gov/pubmed/11286370;

[26] Iamandescu, I.B., (2004), Receptive Music Therapy Psychological and Neurophysiological Premises, Prophylactic and Therapeutic Applications, InfoMedica Publishing House, Bucharest;

[27] Sengewald, B., (1992,1995), Grundaberlegung zur Wirkungweise von Musik, Diplomarbeit Darmstadt;

[28] Ostrander, S., Schroeder, L., Ostrander, N., (2003), Rapid Learning Techniques, Trad. Duna, R. M., Ed. Almatea, Bucharest;

[29] Sacks, O., (2009), Muzicophilia, Tales of Music and The Brain, trad. I. Barbulescu, A., Ed. Humanitas, Bucharest. 